\title{
Multidisciplinary Treatment, Including Locoregional Chemotherapy, for Merkel-Polyomavirus-Positive Merkel Cell Carcinomas: Perspectives for Patients Exhibiting Oncogenic Alternative $\Delta$ exon 6-7 TrkAIII Splicing of Neurotrophin Receptor Tropomyosin-Related Kinase A
}

\author{
Stefano Guadagni ${ }^{1, *(0)}$, Antonietta Rosella Farina ${ }^{1}$, Lucia Annamaria Cappabianca ${ }^{1}$, \\ Michela Sebastiano ${ }^{1}$, Rita Maccarone ${ }^{1}{ }^{(1)}$, Veronica Zelli ${ }^{1}$, Marco Clementi ${ }^{1}(\mathbb{D}$, \\ Alessandro Chiominto ${ }^{1}$, Gemma Bruera ${ }^{1}$ (D), Enrico Ricevuto ${ }^{1}$, Giammaria Fiorentini ${ }^{2}$, \\ Donatella Sarti ${ }^{2}$ and Andrew Reay Mackay ${ }^{1}$ \\ 1 Department of Applied Clinical Sciences and Biotechnology, University of L'Aquila, 67100 L'Aquila, Italy; \\ antonietta.farina@univaq.it (A.R.F.); luciaannamaria.cappabianca@univaq.it (L.A.C.); \\ michela.sebastiano@graduate.univaq.it (M.S.); rita.maccarone@univaq.it (R.M.); \\ veronica.zelli@univaq.it (V.Z.); marco.clementi@univaq.it (M.C.); alessandro.chiominto@univaq.it (A.C.); \\ gemma.gbb@gmail.com (G.B.); enrico.ricevuto@univaq.it (E.R.); andrewreay.mackay@univaq.it (A.R.M.) \\ 2 Department of Onco-Hematology, Azienda Ospedaliera “Ospedali Riuniti Marche Nord”, \\ 61122 Pesaro, Italy; g.fiorentini2020@gmail.com (G.F.); d.sarti@fastwebnet.it (D.S.) \\ * Correspondence: stefano.guadagni@univaq.it
}

Received: 30 September 2020; Accepted: 29 October 2020; Published: 3 November 2020 updates

\begin{abstract}
Merkel cell carcinomas (MCCs) are rare, aggressive, cutaneous neuroendocrine tumours, approximately $80 \%$ of which are caused by the genomic integration of Merkel cell polyomavirus (MCPyV). MCPyV-positive MCCs carry poor prognosis in approximately $70 \%$ of cases, highlighting the need for greater understanding of the oncogenic mechanisms involved in pathogenesis, progression and post-therapeutic relapse, and translation into novel therapeutic strategies. In a previous pilot study, we reported a potential relationship between $M C P y V$ gene expression and oncogenic alternative $\Delta$ exon 6-7 TrkAIII splicing in formalin-fixed paraffin-embedded (FFPE) MCC tissues from a 12-patient cohort of $>90 \%$ MCPyV-positive MCCs, diagnosed at San Salvatore Hospital, L'Aquila, Italy, characterising a new MCC subgroup and unveiling a novel potential MCPyV oncogenic mechanism and therapeutic target. This, however, could not be fully verified due to poor RNA quality and difficulty in protein extraction from FFPE tissues. Here, therefore, we extend our previous observations to confirm the relationship between $\mathrm{MCPyV}$ and oncogenic alternative $\Delta$ exon 6-7 TrkAIII splicing in fresh, nonfixed, MCPyV-positive MCC metastasis by detecting sequence-verified RT-PCR products, including full-length $\Delta$ exon 6-7 TrkAIII, and by Western blot detection of a $100 \mathrm{kDa}$ TrkA protein isoform of identical size to $100 \mathrm{kDa} \Delta$ exon 6-7 TrkAIII expressed by stable transfected SH-SY5Y cells. We also report that in three MCC patients submitted for multidisciplinary treatment, including locoregional chemotherapy, MCPyV large T-antigen mRNA expression, $\triangle$ exon 6-7 TrkAIII mRNA expression and intracellular indirect immunofluorescence (IF) TrkA and phosphorylation protein isoform(s) immunoreactivity in FFPE tissues were not reduced in postchemotherapeutic-relapsed MCCs compared to pretherapeutic MCCs, extending the possible roles of this novel potential $\mathrm{MCPyV}$ oncogenic mechanism from MCC pathogenesis to post-therapeutic relapse and progression. Detection of alternative $\Delta$ exon 6-7 TrkAIII splicing in MCC, therefore, not only characterises a new MCPyV-positive MCC subgroup and unveils a novel potential MCPyV oncogenic mechanism but also identifies patients who may benefit from inhibitors of MCPyV T-antigen and/or TrkAIII expression
\end{abstract}


or clinically approved Trk kinase inhibitors such as larotrectinib or entrectinib, which are known to inhibit activated TrkA oncogenes and to elicit durable responses in TrkA-fusion oncogene-driven cancers, supporting the call for a large-scale multicentre clinical study.

Keywords: Merkel cell carcinoma; Merkel cell polyomavirus; MCPyV T-antigen; tropomyosin tyrosine kinase receptor; $\Delta$ exon 6-7 TrkAIII; isolated pelvic and limb perfusion; larotrectinib; entrectinib

\section{Introduction}

Merkel cell carcinomas (MCCs) are rare, highly aggressive neuroendocrine skin cancers, the incidence of which is increasing in North America, Europe and Australia. Etiological MCC risk factors include Merkel cell polyomavirus (MCPyV) infection, with genomic integration that accounts for approximately $80 \%$ of cases, immunosuppression, ultraviolet (UV)-induced mutation and age greater than 65 years [1-5]. Current first-line treatments for primary and locoregional MCCs include surgical excision with wide margins, followed by radiation therapy (RT) to reduce locoregional recurrence [6]. Recommendations, subject to clinical and/or microscopic lymph node status, also include sentinel lymph node biopsy (SNB) with complete lymph node dissection (CLND) and RT of the nodal basin, where appropriate [6]. Adjuvant RT for MCC is recommended in National Comprehensive Cancer Network (NCCN) guidelines [6] and employed routinely in the USA, although significant differences in regional recurrence rates and overall survival have not been demonstrated [7]. RT is also under consideration for the management of in-transit MCC metastases (stage IIIB) when surgery alone cannot contain the disease or ensure clear margins. However, results for this strategy are inconclusive, nonrandomised and uncontrolled [8].

Current therapeutic strategies for MCCs result in overall 3-year survival rates of approximately $39 \%$, and $26 \%$ to $60 \%$ of patients submitted for surgery, with or without adjuvant RT, represent with local and locoregional recurrences within two years [9]. For patients with advanced locoregional disease, there is currently no specific multidisciplinary therapeutic consensus [10], and patients with stage IV disease are routinely treated with systemic platinum-based etoposide, anthracycline and taxane regimens, alone or in various combinations. Unfortunately, these treatments invariably result in brief responses [11], highlighting the urgent need for alternative therapeutic strategies. Within this context, phase II clinical trials with novel immune checkpoint inhibitors elicit sustained responses in patients with MCPyV- and UV-induced metastatic MCCs [12-14]. Pembrolizumab-programmed cell death protein 1 (PD-1) immune checkpoint inhibitor has been reported to elicit a $56 \%$ response rate in a 26-patient MCC cohort [12] and avelumab-programmed death-ligand 1 (PD-L1) inhibitor a $31.8 \%$ response rate in a heavily pretreated 88-patient MCC cohort, with a higher response rate in patients not previously exposed to chemotherapy [13]. Promising anecdotal antitumor effects have also been reported for the novel targeted vascular endothelial growth factor receptor (VEGFR) tyrosine kinase inhibitors pazopanib (4 patients) and cabozantinib (1 patient) [15] for direct targeting of MCC cell surface antigens, for an antibody raised against the epithelial cell adhesion molecule-1/cluster of differentiation CD326, and for the cytokines tumour necrosis factor (TNF) and gamma interferon (INF $\gamma$ ) [16]. In contrast, the combination of surgical intervention with locoregional chemotherapeutic procedures has largely been overlooked for treating locoregionally advanced MCCs, with or without distant metastases, despite routine use over the past 20 years as integrated approaches for local disease control and limb preservation in highly specialised American [17-21], European [22-24] and Australian [25] centres.

Locoregional chemotherapeutic procedures include (i) isolated limb infusion (ILI) [19-21,25], (ii) isolated limb perfusion (ILP) [17-19,22,23], and (iii) isolated pelvic and limb perfusion (IPLP) for patients with pelvic and/or inguinal involvement (this study and [24]). All three procedures incorporate technologically controlled extracorporeal blood circulation. ILP is performed under conditions of 
oxygenation, high flow rates (150-1000 $\mathrm{mL} / \mathrm{min}$ ), circuit hyperthermia to maintain tissue normothermia and requires specialised surgical skill, whereas ILI and IPLP are executed under conditions of hypoxia, low flow-rates (50-150 $\mathrm{mL} / \mathrm{min}$ ) and mild circuit hyperthermia to maintain tissue normothermia, with the option of chemofiltration, and can be performed not only by surgeons but also percutaneously by interventional radiologists $[26,27]$. In a literature review and despite the absence of standardised therapeutic strategies, 16 ILP and 16 ILI cases in the USA [18,25] and 10 IPL and 3 IPLP cases in Europe (this study and [23,24]) have all employed the nitrogen mustard-like, DNA-alkylating chemotherapeutic agent Melphalan (this study and [18,23-25]) In these studies, ILP elicited an impressive 90\% overall response rate, associated with 5 months median progression-free survival and 50 months median overall survival $[18,23]$, which was comparable to the approximately $80 \%$ overall response rate elicited by ILI, reported without median progression-free survival and overall survival data $[18,25]$. This suggests that the ILI procedure, which is less complex and associates with fewer complications, should be the preferred procedure for local disease control and limb preservation in advanced-stage limb-localised MCC [25]. On the other hand, IPLP is currently the only appropriate procedure for the locoregional chemotherapeutic management of advanced-stage MCCs that are localised to pelvic and inguinal regions. However, there have been only two reports of this procedure (this study and [24]).

MCC diagnosis, however, continues to carry poor prognosis in spite of these advances, highlighting the need for a further understanding of oncogenic mechanisms involved in this rare tumour type and translation into novel therapeutic strategies. Within this context, in a recent pilot study of MCC FFPE tissues, we reported a novel potential MCPyV oncogenic mechanism, characterised by the promotion of oncogenic alternative $\Delta$ exon 6-7 TrkAIII mRNA splicing of the tropomyosin-related tyrosine kinase receptor TrkA [28]. Oncogenic alternative $\Delta$ exon 6-7 TrkAIII mRNA splicing was originally identified in advanced-stage primary human neuroblastomas (NBs), results in expression of the $\Delta$ exon 6-7 TrkAIII oncoprotein that transforms NIH3T3 mouse embryo fibroblasts and exhibits oncogenic activity in a variety of in-vitro and in-vivo models $[29,30]$. Furthermore, analysis of potential promoters of alternative $\Delta$ exon 6-7 TrkAIII splicing in NB cells identified the polyomavirus simian virus 40 (SV40) large T-antigen [30], raising the possibility that closely related polyomavirus MCPyV T-antigens may also promote alternative $\Delta$ exon 6-7 TrkAIII splicing in MCPyV-positive MCCs. This hypothesis was supported in our pilot study [28], which detected a close relationship between MCPyV T-antigen expression and alternative $\Delta$ exon 6-7 TrkAIII splicing in FFPE MCC tissues, characterising a new MCC subset and identifying a novel potential MCPyV oncogenic mechanism and therapeutic target (Figure 1). This, however, could not be fully verified due to the poor RNA quality and difficulty in protein extraction from FFPE tissue [28].

In the present study, therefore, we extend our previous report supporting the use of FFPE MCC tissue for MCPyV-positive MCC diagnosis, retrospective study and molecular analysis to confirm the association between MCPyV T-antigen and oncogenic alternative $\Delta$ exon 6-7 TrkAIII splicing in fresh nonfixed MCPyV-positive metastatic MCC. We also extend the potential role of this novel oncogenic mechanism from MCC pathogenesis to post-therapeutic relapse and progression and propose that its detection may identify MCC patients who could benefit from novel MCPyV T-antigen and TrkAIII inhibitory therapeutic strategies. 


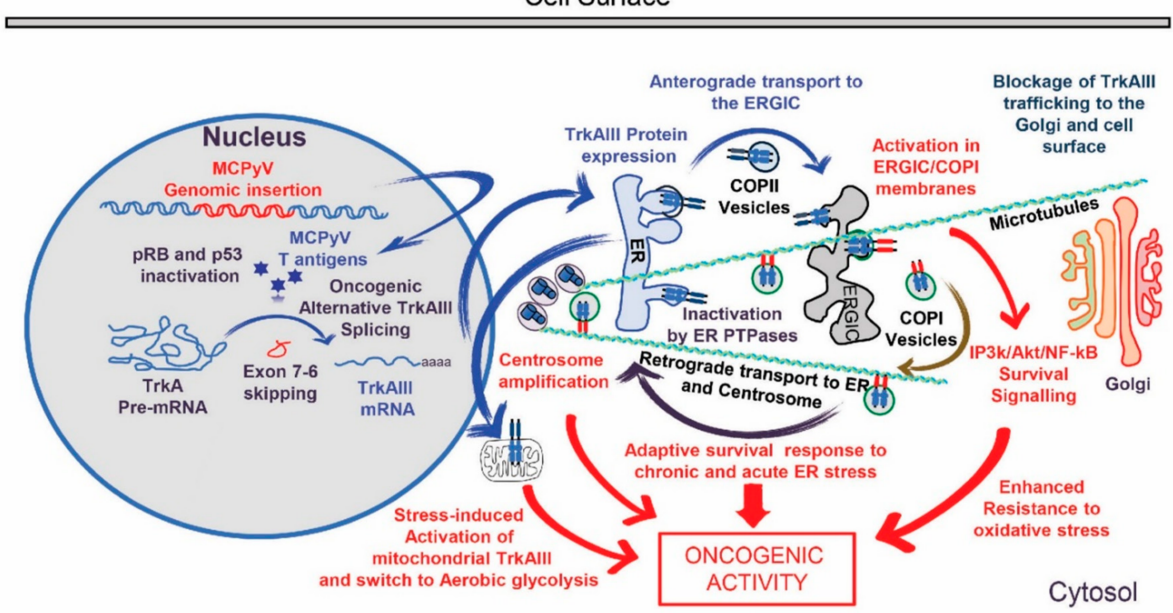

Figure 1. Schematic representation of genomic Merkel cell polyomavirus (MCPyV) insertion, T-antigen expression and induction of oncogenic alternative $\Delta$ exon 6-7 TrkAIII splicing in Merkel cell carcinoma (MCC), leading to spontaneous intracellular $\Delta$ exon 6-7 TrkAIII activation within endoplasmic reticulum intermediate and COP1 vesicle membrane compartments (ERGIC/COP1), inhibition of anterograde $\Delta$ exon 6-7 TrkAIII transport, retrograde-activated $\Delta$ exon 6-7 TrkAIII transport back to the endoplasmic reticulum (ER), centrosome and mitochondria, and oncogenic activity characterised by inositol-triphosphate 3-kinase (IP3K)/Akt survival signalling, centrosome amplification, an adaptive survival ER-stress response, enhanced resistance to oxidative stress and stress-induced aerobic glycolysis.

\section{Results}

\subsection{Clinical Data}

From a total of 7175 skin cancers, 12 MCCs $(0.17 \%)$ spanning the period 2009-2019, histologically diagnosed by immunoreactivity for neural cell adhesion molecule (CD56), cytokeratin 20 and cytokeratin AE1/AE3 were selected from the San Salvatore Hospital Department of Pathology database (L'Aquila, Italy). The median age was 73.5 years (interquartile range (iqr) $=60.5-82.5$ ), and the clinical characteristics of the cohort are reported in Table 1. The median overall survival time following multidisciplinary treatment and from diagnosis was 26 months (iqr $=21-35) ; 10$ patients treated prior to 2019 were dead by February 2020, and 2 patients were still alive at 13 and 5 months from the time of diagnosis, with the disease. All 12 patients underwent surgical excision in multidisciplinary treatment regimes, which included subsequent RT in 2 cases, Melphalan IPLP in 3 cases (Figure 2A), systemic chemotherapy in 2 cases and immune checkpoint inhibitor therapy in 2 cases. One patient, who presented with chronic neutropenia and received concomitant sofosbuvir/daclastavir therapy for $\mathrm{HCV}$ infection [24], experienced an unexpected 56-month response to multidisciplinary treatment, consisting of surgical excision followed by melphalan IPLP (Table 2). Approximately one year following the cessation of antiviral therapy, this patient developed thigh recurrences and was treated with avelumab PD-L1 inhibitory monoclonal antibody [14] in accordance with multidisciplinary board suggestions, but failed to respond (Figure 2B).

Two other patients with stage IV distant metastatic disease were subjected to surgical excision combined with IPLP. Both patients experienced brief 3- and 7-months of control of local disease, followed by local progression, and were subsequently treated with systemic chemotherapy but failed to respond (Table 2). 
Table 1. Clinical characteristics of Merkel cell carcinoma (MCC) patients.

\begin{tabular}{|c|c|}
\hline Clinical Characteristics & $\mathbf{N}(\%)$ \\
\hline \multicolumn{2}{|l|}{ Gender } \\
\hline Female & $7(58.3)$ \\
\hline Male & $5(41.7)$ \\
\hline \multicolumn{2}{|l|}{ Age (Years) } \\
\hline$\leq 65$ & $4(33.3)$ \\
\hline$>65$ & $8(66.7)$ \\
\hline \multicolumn{2}{|c|}{ Primary Location } \\
\hline Head and/or Neck & $2(16.7)$ \\
\hline Trunk & $4(33.3)$ \\
\hline Extremities & $6(50.0)$ \\
\hline \multicolumn{2}{|c|}{ Stage (AJCC 2019) at Molecular Analysis } \\
\hline I & $2(16.7)$ \\
\hline II A & $4(33.3)$ \\
\hline II B & $1(8.3)$ \\
\hline III B & $3(25.0)$ \\
\hline IV & $2(16.7)$ \\
\hline \multicolumn{2}{|c|}{ Multidisciplinary Treatment } \\
\hline Surgical excision & $12(100.0)$ \\
\hline Radiation therapy & $2(16.7)$ \\
\hline Melphalan IPLP & $3(25.0)$ \\
\hline Systemic chemotherapy & $2(16.7)$ \\
\hline Immune checkpoint inhibitors & $2(16.7)$ \\
\hline \multicolumn{2}{|l|}{ Status } \\
\hline Dead & $10(83.3)$ \\
\hline Alive & $2(16.7)$ \\
\hline
\end{tabular}

\subsection{Molecular Data}

RT-PCR detected MCPyV VP-1, small t-antigen and large T-antigen mRNA expression in RNAs from 11 of 12 FFPE MCC tissues, confirming a > 90\% MCPyV-positive MCC cohort (Table 2). RNAs exhibiting MCPyV large T-antigen expression also generated sequence-verified RT-PCR products, consistent with alternative $\Delta$ exon 6-7 TrkAIII mRNA splicing, that were not detected in the MCPyV T-antigen-negative FFPE MCC (this study and [28]). Indirect IF analysis of MCPyV-positive FFPE MCC tissues also detected IF immunoreactivity for intracellular TrkA protein isoforms and variable immunoreactivity for tyrosine-phosphorylated TrkA isoforms, consistent with potential intracellular $\Delta$ exon 6-7 TrkAIII protein expression and activation (Table 3). In an important extension of our previous study, we were fortunate to obtain FFPE MCC tissues from three of the original 12 patients of the cohort, which permitted a comparison of the influence of melphalan IPLP chemotherapy on MCPyV T-antigen and $\Delta$ exon 6-7 TrkAIII expression. In this comparison, no significant differences were detected in the mean $( \pm \mathrm{SD}$ ) densitometric ratios of MCPyV T-antigen or $\Delta$ exon 6-7 TrkAIII to $18 \mathrm{~S}$ rRNA RT-PCR, employed as a stable internal control for relative RT-PCR analysis (Figure 3), and IF immunoreactivity for phosphorylated TrkA isoform(s) in MCCs that relapsed following melphalan IPLP chemotherapy was, if anything, enhanced rather than reduced (Figure 3), supporting a potential role for this oncogenic mechanism in post-therapeutic disease progression. 
A

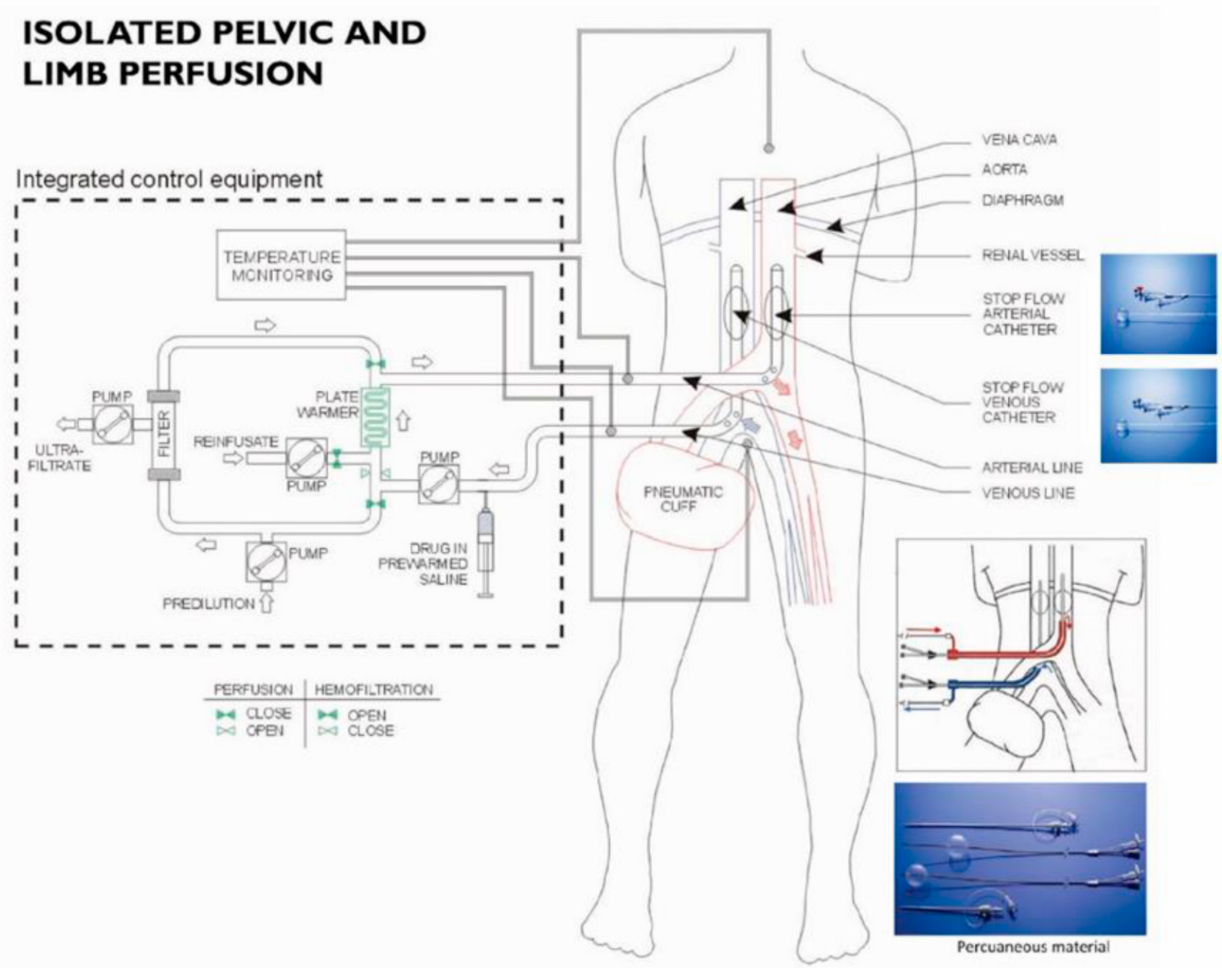

B

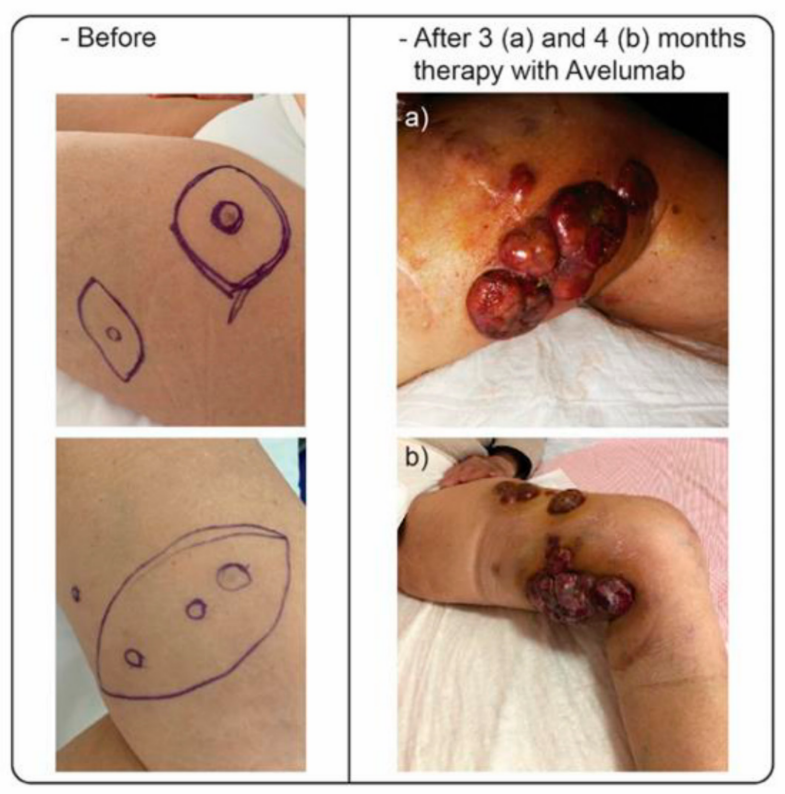

Figure 2. (A) Schematic representation of isolated pelvic and limb perfusion with extracorporeal blood circulation controlled by an integrated equipment circuit, complete with chemofiltration unit and apparatus used for open and percutaneous approaches (cartouche). (B) Response to avelumab therapy: MCC tumours prior to avelumab therapy (left panels) and following (a) 3 months and (b) 4 months of avelumab therapy (right panels). 
Table 2. Clinical characteristics of three advanced-stage MCC patients submitted for locoregional chemotherapy during multidisciplinary treatment.

\begin{tabular}{|c|c|c|c|c|c|c|}
\hline $\begin{array}{l}\text { (A) Patient Id } \\
\text { (B) Primary Site } \\
\text { (C) Recurrence } \\
\text { Location/Age } \\
\text { (Years)/Stage }\end{array}$ & $\begin{array}{l}\text { (A) Previous } \\
\text { Therapy } \\
\text { (B) Concomitant } \\
\text { Disease (Therapy) }\end{array}$ & $\begin{array}{l}\text { 1st Treatment } \\
\text { Distant } \\
\text { Disease Status }\end{array}$ & $\begin{array}{c}\text { 2nd Treatment } \\
\text { Distant Disease Status }\end{array}$ & $\begin{array}{c}\text { 3rd Treatment } \\
\text { Distant Disease Status }\end{array}$ & $\begin{array}{l}\text { (A) Concomitant Therapy } \\
\text { Before Progression } \\
\text { (B) PFS from 1st Locoregional } \\
\text { Treatment } \\
\text { (C) Progression Site } \\
\text { (D) Therapy at Progression }\end{array}$ & $\begin{array}{c}\text { Censor } \\
\text { (February } \\
2020) \\
\text { OS from 1st } \\
\text { Locoregional } \\
\text { Treatment }\end{array}$ \\
\hline $\begin{array}{c}\text { (A) } 1 \\
\text { (B) Calcaneal region } \\
\text { (C) Groin and limb } \\
\text { /73/IIIB } \\
\text { (pathological) }\end{array}$ & $\begin{array}{l}\text { (A) WSE; SNB; CLND } \\
\text { Chronic Neutropenia } \\
\text { (B) Hepatitis C } \\
\text { (Sofosbuvir/ } \\
\text { Daclastavir) }\end{array}$ & $\begin{array}{c}\text { WSE } \\
\text { (10 nodules }>1 \mathrm{~cm} \text { diameter }) \\
\text { IPLP }\end{array}$ & $\begin{array}{c}\text { WSE } \\
\text { (3 nodules }<1 \mathrm{~cm} \text { diameter) } \\
\text { IPLP }\end{array}$ & $\begin{array}{c}\text { WSE } \\
\text { (1 nodule }<1 \mathrm{~cm} \text { diameter }) \\
\text { IPLP }\end{array}$ & $\begin{array}{l}\text { (A) Sofosbuvir/ } \\
\text { Daclastavir } \\
\text { (24 months) } \\
\text { (B) } 56 \text { months } \\
\text { (C) Locoregional } \\
\text { (D) Avelumab }\end{array}$ & $\begin{array}{c}\text { Dead } \\
59 \text { months }\end{array}$ \\
\hline $\begin{array}{l}\text { (A) } 2 \\
\text { (B) Anterior abdomen } \\
\text { (C) Pelvis and limb } \\
\text { plus distant/58/IV } \\
\text { (clinical) }\end{array}$ & $\begin{array}{l}\text { (A) WSE } \\
\text { and RT }\end{array}$ & $\begin{array}{c}\text { WSE } \\
\text { (7 nodules }>2 \mathrm{~cm} \text { diameter) } \\
\text { IPLP } \\
\text { SDi in bone metastases }\end{array}$ & $\begin{array}{c}\text { WSE } \\
\text { ( } 5 \text { nodules }>2 \mathrm{~cm} \text { diameter) } \\
\text { IPLP } \\
\text { SDi in bone metastases }\end{array}$ & $\begin{array}{c}\text { WSE } \\
\text { (4 nodules }>2 \mathrm{~cm} \text { diameter) } \\
\text { IPLP } \\
\text { SDi in bone metastases }\end{array}$ & $\begin{array}{l}\text { (B) } 7 \text { months } \\
\text { (C) Locoregional and distant } \\
\text { (D) Platinum-based systemic } \\
\text { chemotherapy until dead }\end{array}$ & $\begin{array}{c}\text { Dead } \\
12 \text { months }\end{array}$ \\
\hline $\begin{array}{l}\text { (A) } 3 \\
\text { (B) Gluteal region } \\
\text { (C) Pelvis and limb } \\
\text { plus distant/75/IV } \\
\text { (clinical) }\end{array}$ & $\begin{array}{c}\text { (A) WSE } \\
\text { (B) Heart arrhythmia } \\
\text { (Coumadin); } \\
\text { Glaucoma } \\
\text { (Propanolol) }\end{array}$ & $\begin{array}{l}\text { WGSE/CLND (5 metastatic lymph nodes) } \\
\text { IPLP } \\
\text { SDi in lung, liver and brain metastases }\end{array}$ & $\begin{array}{c}\text { IPLP alone } \\
\text { SDi in lung, liver and brain } \\
\text { metastases }\end{array}$ & & $\begin{array}{c}\text { (A) Coumadin/ } \\
\text { Propanolol } \\
\text { (B) } 3 \text { months } \\
\text { (C) Locoregional and distant } \\
\text { (D) Platinum-based systemic } \\
\text { chemotherapy for } 23 \text { months }\end{array}$ & $\begin{array}{l}\text { Dead } \\
30 \text { months }\end{array}$ \\
\hline
\end{tabular}

biopsy; CLND, complete lymph node dissection; SDi, stable disease; RT, radiotherapy. 
Table 3. Molecular characteristics of MCC patients.

\begin{tabular}{cc}
\hline MCC Molecular Characteristics. & N (\%) \\
\hline MCPyV Gene Expression & \\
\hline Yes & $11(91.7)$ \\
No & $1(8.3)$ \\
\hline MCPyV Large T-Antigen & \\
\hline Present & $11(91.7)$ \\
High & $10(83.4)$ \\
Moderate & $1(8.3)$ \\
Negative & $1(8.3)$ \\
\hline TrkA Expression & $1(8.3)$ \\
\hline High & $4(33.3)$ \\
Moderate & $7(58.4)$ \\
Low & \\
\hline TrkAIII Expression & $8(66.7)$ \\
\hline High & $3(25.0)$ \\
Moderate & $1(8.3)$ \\
Low & $1(8.3)$ \\
\hline Phosphorylated TrkA/TrkAIII IF \\
\hline High. & $6(50.0)$ \\
Moderate & $2(16.7)$ \\
Negative & \\
\hline
\end{tabular}

In our previous study [28], the relationship between MCPyV and oncogenic alternative $\Delta$ exon 6-7 TrkAIII splicing, detected in FFPE MCC tissues, could not be fully verified due to poor RNA quality and difficulty in protein extraction [31]. Here, we present evidence that confirms this relationship in tissue from fresh nonfixed MCPyV-positive MCC metastasis that subsequently became available from one patient in this cohort. RT-PCR of undegraded RNAs from this metastatic MCC detected: (i) MCPyV VP1, small t-antigen and large T-antigen mRNA expression confirming an MCPyV-positive diagnosis (Figure 4A); (ii) a 2372-bp (base pair) product expected for full length fully spliced TrkA and a 2096-bp product expected for full length alternatively spliced $\Delta$ exon 6-7 TrkAIII, using primers spanning Trk $A$ exons 1 to 17; (iii) a 1112-bp product expected for fully spliced exon 1-8 TrkA and an 836-bp product expected for $\Delta$ exon 6-7 TrkAIII, using primers spanning TrkA exons 1 to 8; (iv) a 139-bp product expected for $\Delta$ exon 6-7 TrkAIII using the TrkAIII-specific primer set; (v) a single 1280-bp product expected for fully spliced exons 10 to17 TrkA, using primers spanning TrkA exons 10-17 (Figure 4B). The 1112-bp and 836-bp exon 1-8 RT-PCR products were gel-purified and further characterised as representing fully spliced TrkA and alternatively spliced $\Delta$ exon 6-7 TrkAIII, respectively, by RT-PCR using TrkA and $\Delta$ exon 6-7 TrkAIII-specific primers (Figure 4 C) and by detection of exons 6, 7 and 8 sequences in the 1112-bp fully spliced TrkA product (not shown) and the novel exon 5-8 splice junction in the 836-bp $\Delta$ exon 6-7 TrkAIII product (Figure 4D), identical to the original TrkAIII sequence (29) deduced from TrkAI splice variant reference sequence NM_001012331.2. Additional RT-PCR products generated using primers spanning TrkA exons 1 to 8 included a major 500-bp product (Figure 4B), which was also gel-purified (Figure 4C) and sequence-characterised as a novel alternative $\Delta$ exon 2-7 TrkAIV splice variant exhibiting cassette exons 2 to 7 skipping, coding for 3 frame-shift-induced stop codons initiating at 40 codons downstream of the novel exon 1-8 splice junction (Figure 4C,D). 


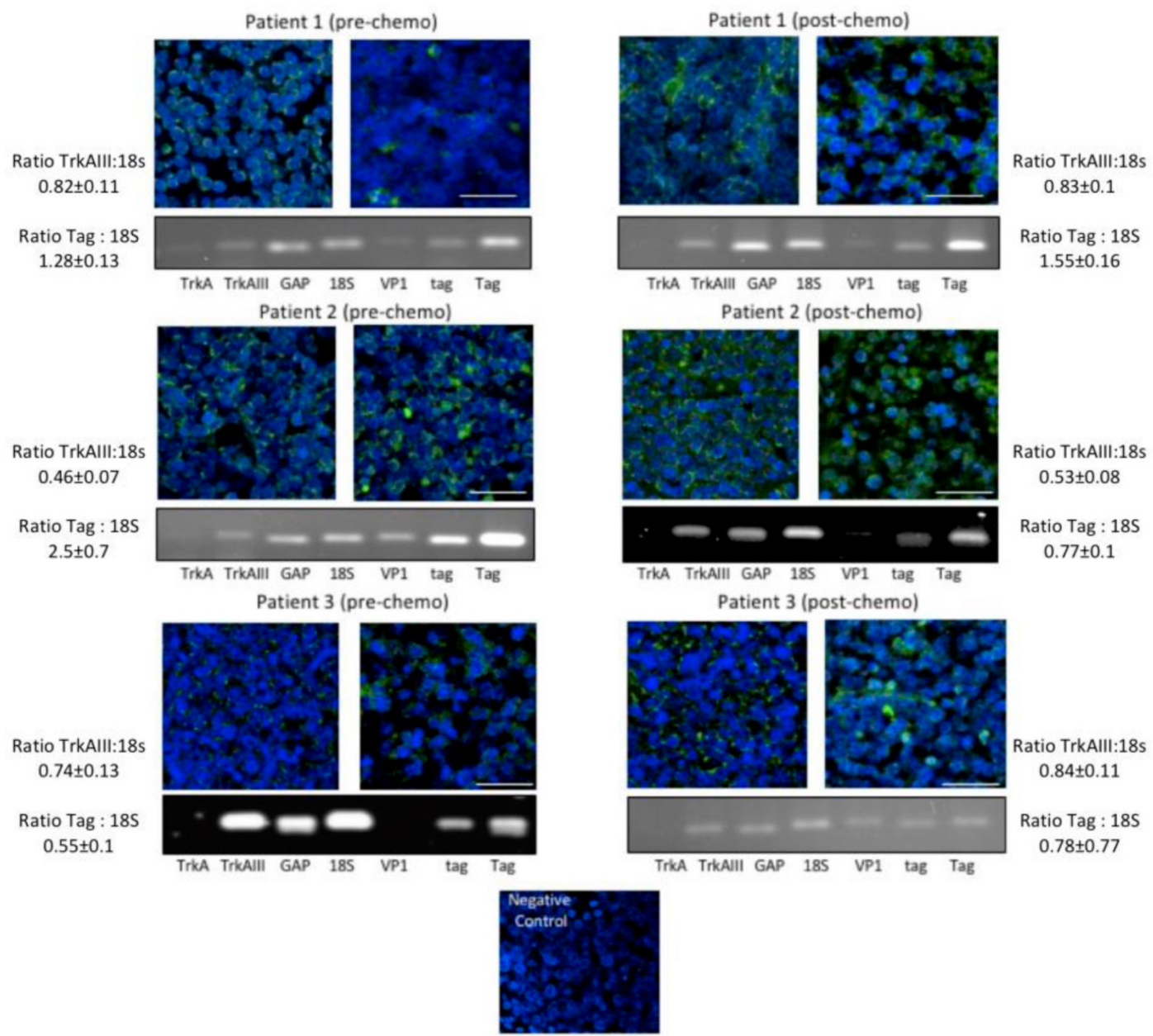

Figure 3. Representative indirect immunofluorescence (IF) micrographs demonstrating immunoreactivity to anti-TrkA and anti-Y490 phosphorylated TrkA antibodies in $5 \mu \mathrm{m}$ formalin-fixed paraffin-embedded (FFPE) sections of primary MCCs from 3 patients prior to chemotherapy (prechemo) and postchemotherapy (postchemo) recurrent MCCs from the same patients $(\mathrm{bar}=50 \mu \mathrm{m})$. Negative control represents MCC FFPE tissue from Patient 3 incubated with preimmune rabbit IgG and secondary FITC conjugated anti-rabbit antibody. Under each set is a representative agarose gel demonstrating RT-PCR levels of TrkA, TrkAIII, glyceraldehyde 3-phosphate dehydrogenase (GAP), 18S RNA, MCPyV viral capsid protein (VP1), MCPyV small $\mathrm{t}$-antigen (tag) and MCPyV large t-antigen (Tag) in RNAs purified from $50 \mu \mathrm{m}$ serial sections of the same FFPE MCC tissues. The densitometric ratios $( \pm \mathrm{SD})$ of TrkAIII to $18 \mathrm{~S}$ rRNA (TrkAIII: 18S) and MCPyV Tag to $18 S$ rRNA (Tag: 18S) RT-PCR products are provided for each patient.

In addition to RT-PCR, Western blots with an antibody that recognises both fully-spliced TrkA and $\Delta$ exon 6-7 TrkAIII $[28,29]$ detected a $100-\mathrm{kDa}$ immunoreactive species in protein extracts $(100 \mu \mathrm{g})$ from the fresh metastatic MCC, not detected in an extract of normal skin (100 $\mu \mathrm{g})$, of identical size to 100-kDa $\Delta$ exon 6-7 TrkAIII detected in stable $\Delta$ exon 6-7 TrkAIII transfected SH-SY5Y cell extracts but not in either stable TrkA-transfected SH-SY5Y cell extracts, which contained 140-kDa fully spliced TrkA or in stable empty pcDNA vector-transfected SH-SY5Y cell extracts used as a negative control (20 $\mu \mathrm{g})$ [29] (Figure 4E), consistent with $\Delta$ exon 6-7 TrkAIII protein expression in the fresh MCPyV-positive metastatic MCC. 
A

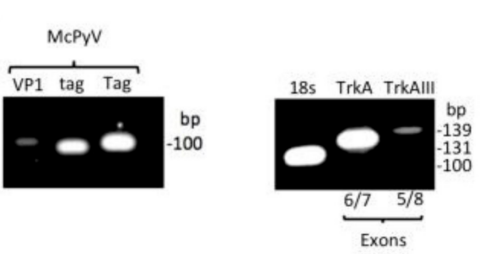

B

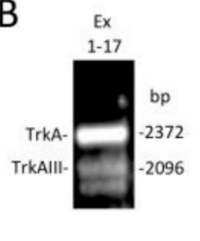

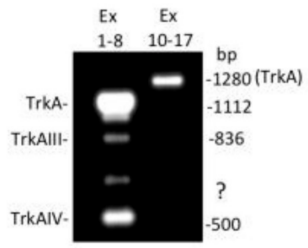

\section{C}
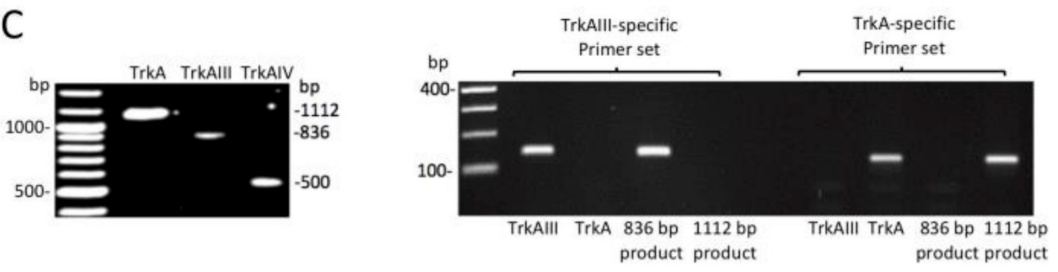

D
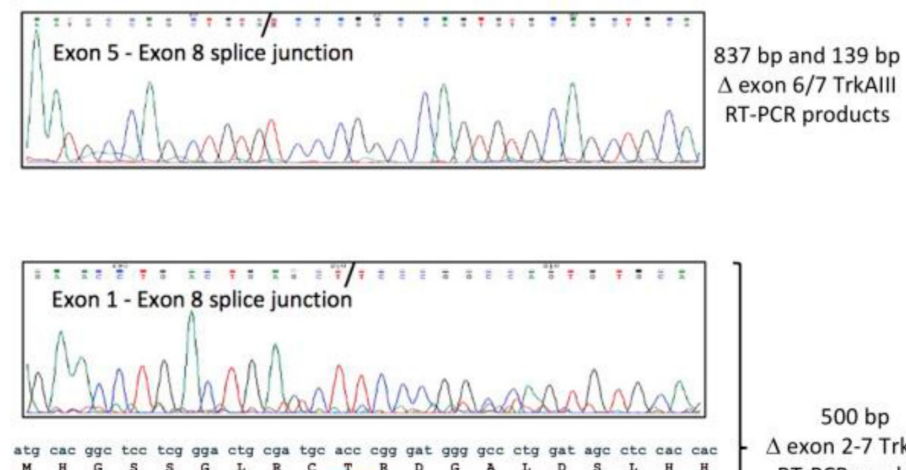

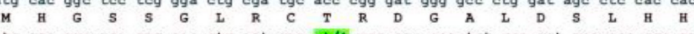
ctg cec ggc gca gag aac ctg act gag ct/t cec ggc cag tgt gca gct gca cac ggc ggt gga gat gea eca etg gtg eat eec ett ote tgt gga tgg gea gee gge ace gte tet geg

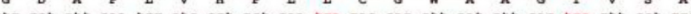
L $\underset{A}{g c c}$ ggc agc caa tga gac ogt geg gca cgg gtg tet gcg cet caa cea gce cac cea cgt caa caa cgg caa cta cac gct

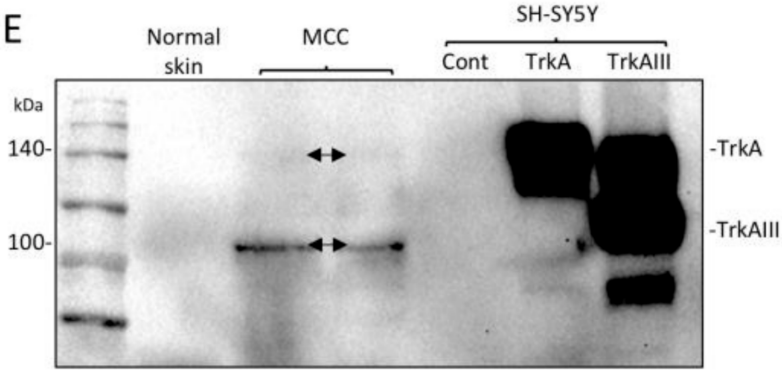

Figure 4. (A) RT-PCR demonstrating MCPyV VP1, small t-antigen (tag) and large T-antigen (Tag) (left panel), and 18s rRNA, exon 6 and 7 containing (exons 6/7 TrkA) and noncontaining (exons 5/8, TrkAIII) products generated from metastatic MCPyV-positive MCC RNA. (B) RT-PCR demonstrating (left panel) a 2372-bp product expected for full-length fully spliced TrkA and a 2096-bp product expected for $\Delta$ exon 6-7 TrkAIII generated from metastatic MCC RNA using primers spanning TrkA exons 1 to 17, and (right panel) a 1112-bp product expected for fully spliced TrkA exons 1-8, an 836-bp product expected from $\Delta$ exon 6-7 TrkAIII exons 1-8, a yet to be characterised product (?) and the 500-bp product characterised as $\Delta$ exon 2-7 TrkAIV, generated from metastatic MCPyV-positive MCC RNA, using primers spanning TrkA exons 1 to 8 , plus a unique 1280-bp product expected for fully spliced TrkA exons 10-17, generated from metastatic MCPyV-positive MCC RNA using primers spanning TrkA exons 10 to 17. (C) Gel-purified 1112-bp (fully spliced TrkA), 836-bp ( $\Delta$ exon 6-7 TrkAIII) and 500-bp ( $\Delta$ exon 2-7 TrkAIV) TrkA exon 1 to 8 RT-PCR products (left panel), and (right panel) RT-PCR confirmation 
of $\Delta$ exon 6-7 TrkAIII and TrkA identity of TrkA and D exon 6-7 TrkAIII control cDNAs, gel-purified 1112-bp TrkA exon 1-8 and 836-bp $\Delta$ exon 6-7 TrkAIII RT-PCR products, using TrkA and $\Delta$ exon 6-7 TrkAIII-specific primers. (D) Sequence confirmation of the novel $\Delta$ exon 6-7 TrkAIII exon 5/8 splice junction in the gel-purified 836-bp $\Delta$ exon 6-7 TrkAIII exon 1-8 RT-PCR product (3rd panel), and confirmation of the novel exon $1 / 8$ splice junction (green) in the 500-bp $\Delta$ exon $2-7$ TrkAIV exon 1-8 RT-PCR product (4th panel), plus a section of nucleotide and potential amino acid sequence for $\Delta$ exon 2-7 TrkAIV demonstrating frame-shift induced tga stop codons (red). (E) Western blot demonstrating $140 \mathrm{kDa}$ and $100 \mathrm{kDa}$ (arrows) TrkA immunoreactive species in protein extracts from the MCPyV-positive metastatic MCC (MCC) but not in a normal skin extract (100 $\mu \mathrm{g}$ loads), compared to $140 \mathrm{kDa}$ fully spliced TrkA and $100 \mathrm{kDa} \Delta$ exon 6-7 TrkAIII in stable TrkA and $\Delta$ exon 6-7 TrkAIII transfected SH-SY5Y cell extracts but not in stable empty pcDNA vector-transfected SH-SY5Y cell extract negative control (Cont; $20 \mu \mathrm{g}$ loads).

\section{Discussion}

Rare, highly aggressive neuroendocrine MCC skin cancers invariably carry a poor prognosis, which combined with a general lack of specific multidisciplinary therapeutic consensus for the treatment of advanced locoregional and stage IV disease, highlights the great need for improved understanding of the oncogenic mechanisms involved in MCC pathogenesis, post-therapeutic relapse and progression, and translation into novel therapeutic strategies.

Here, we present an extension of a previous pilot study [28] that confirms the importance of FFPE MCC tissues as a source of rare tumour material for MCPyV-positive MCC diagnosis, retrospective study and molecular investigation of novel potential oncogenic mechanisms. We also confirm the association between $\mathrm{MCPyV}$ gene expression and oncogenic alternative $\Delta$ exon 6-7 TrkAIII splicing in a fresh nonfixed metastatic MCC and extend the potential roles of MCPyV-driven oncogenic alternative $\Delta$ exon 6-7 TrkAIII splicing in MCC from pathogenesis to postchemotherapeutic relapse and progression. Whilst this genetic association cannot currently be considered causative, our observations focus attention on the therapeutic possibility of targeting this novel potential oncogenic mechanism in MCC.

The importance of FFPE tissues as a source of rare tumour material for retrospective study and molecular analysis is contrasted by the poor quality of RNA and difficulty in extracting proteins from FFPE tissues, making analysis and interpretation of gene expression difficult [31]. Previously, we reported RT-PCR that MCPyV T-antigen mRNA expression was frequently associated with oncogenic alternative $\triangle$ exon 6-7 TrkAIII splicing in FFPE MCC tissues, characterising a new MCPyV-positive MCC subgroup and identifying a novel potential MCPyV oncogenic mechanism [28]. This was based upon detecting small $(<200 \mathrm{bp}$ ) sequence-characterised $\Delta$ exon 6-7 TrkAIII RT-PCR products and by indirect IF evidence supporting potential intracellular $\Delta$ exon 6-7 TrkAIII TrkA protein expression and activation. However, this could not be fully verified by additional RT-PCR or Western blotting due to problems with FFPE tissues [28]. Here, we present evidence that confirms the association between $M C P y V$ gene expression and oncogenic alternative $\Delta$ exon 6-7 TrkAIII splicing in a fresh nonfixed MCPyV-positive MCC metastasis that became available from one patient in the cohort, extending the possible roles of this potential oncogenic mechanism to metastatic disease. RT-PCR of this metastatic MCPyV-positive MCC detected expected products for both full-length fully spliced TrkA and full-length alternatively spliced $\Delta$ exon 6-7 TrkAIII, with the 1112-bp exon 1-8 fully spliced TrkA and 836-bp exon 1-8 and 139-bp $\Delta$ exon 6-7 TrkAIII RT-PCR products confirmed by DNA sequence comparison to the TrkAI splice variant reference sequence NM_001012331.2, confirming alternative $\Delta$ exon 6-7 TrkAIII mRNA splicing. Furthermore, the unique 1280-bp TrkA exons 10 to 17 RT-PCR product, expected for fully spliced TrkA, confirms that alternative TrkA splicing in this metastatic MCPyV-positive MCC involved TrkA exons 1-8 but not exons 10-17. Furthermore, Western blots also detected a 100-kDa TrkA isoform, consistent with $\Delta$ exon 6-7 TrkAIII protein, in protein extracts from the metastatic MCC, which was not detected in a normal skin extract and was of an identical molecular size to the 100-kDa $\Delta$ exon 6-7 TrkAIII expressed by stable $\Delta$ exon 6-7 TrkAIII-transfected 
SH-SY5Y cells compared to 140-kDa fully spliced TrkA expressed by stable TrkA-transfected SH-SY5Y cells pcDNA-transfected SH-SY5Y cells (negative control) [29]. An additional and prominent RT-PCR product was also detected in metastatic MCC RNA and was characterised as a novel $\Delta$ exon 2-7 TrkAIV splice exhibiting cassette exon 2-7 skipping, resulting in the introduction of 3 frame shift-induced stop codons downstream of the novel exon 1-8 splice junction. We are currently investigating whether this is expressed as a truncated protein and/or exhibits long noncoding RNA functions.

In addition, we also report comparative molecular and indirect IF analysis of FFPE MCC tissues from three patients, obtained prior to and following melphalan- or platinum-based chemotherapy. This comparison did not detect any reductions in the constitutive pretreatment levels of either MCPyV large T-antigen mRNA expression, $\Delta$ exon 6-7 TrkAIII mRNA expression or TrkA and phosphorylated TrkA isoform(s) IF immunoreactivity in tumours that relapsed following treatment. Although this does not prove a causative role for $\Delta$ exon 6-7 TrkAIII splicing in treatment resistance, we have previously shown that $\Delta$ exon 6-7 TrkAIII enhances NB cell resistance to a variety of agents, including cisplatin [30], suggesting the possibility that, in addition to playing potential oncogenic roles in MCC pathogenesis and metastatic progression, alternative $\Delta$ exon 6-7 TrkAIII splicing may also promote therapeutic resistance and postchemotherapeutic relapse in this tumour type, further focusing attention on the therapeutic possibility of targeting this potential mechanism in MCPyV-positive MCC.

Of additional interest, one patient with stage IIIB MCPyV-positive MCC experienced an unexpected 56-month response duration following surgical tumour excision and IPLP locoregional therapy [24]. This patient presented with chronic neutropenia and received concomitant sofosbuvir/daclastavir therapy for Hepatitis $\mathrm{C}$ infection, suggesting that sofosbuvir/daclastavir therapy may have inadvertently and indirectly inhibited the oncogenic activity $\mathrm{MCPyV}$, rendering disseminated MCC cells that survived locoregional IPLP dormant for the duration of antiviral therapy. This patient presented with relapsed disease approximately 1 year after the suspension of antiviral therapy and was treated with avelumab PD-L1 checkpoint inhibitor but failed to respond (Figure 2B) and subsequently died, suggesting that immune checkpoint inhibitor efficacy should also be re-evaluated in patients presenting with altered immune/inflammatory responses [32] and in patients with stage IIIB, in addition to stage IV, disease.

Limitations of this study include the small retrograde sample size explained by the relative rarity of MCC, the poor quality of RNAs and the impracticability of protein extraction from FFPE tissues, limiting accurate gene expression evaluation, addressed in this study using a fresh nonfixed MCPyV-positive MCC metastasis, and the fact that this is a single-centre study due to the lack of specialised centres that perform surgery with IPLP and provide tissues for molecular analyses.

\section{Patients and Methods}

\subsection{Patients}

This STROCSS-criteria-compliant [33] retrospective cohort study was approved by the Ethics committee of ASL n.1, Abruzzo, Italy (Chairperson: G. Piccioli; protocol number10/CE/2018 n.1419). All patients provided fully informed written consent, including the publication of scientific images. The 12 MCC skin cancers in this study were histologically diagnosed from 2009 to 2019, selected from the database of the Department of Pathology San Salvatore Hospital, L'Aquila, and the clinical characteristics are reported in Table 1 and represent the same patient cohort reported previously in our pilot study [28].

\subsection{Clinical Methods}

Multidisciplinary treatments included surgical oncological excision in all 12 cases, followed by RT in 2 patients, locoregional IPLP chemotherapy in 3 patients, systemic chemotherapy in 2 patients, and immune checkpoint inhibitor therapy in 2 patients. Surgical and percutaneous IPLP procedures (Figure 2A) were performed under general anaesthesia and are detailed elsewhere [24,26,27]. Melphalan was used at a dose of $30 \mathrm{mg} / \mathrm{m}^{2}$. 


\subsection{Molecular Methods}

MCPyV VP-1, large T-antigen and small t-antigen mRNA expression, as an index of MCPyV infection and genomic integration, and TrkA isoform mRNA expression were assessed by RT-PCR, using gene-specific primers to generate RT-PCR products of <200-bp for MCPyV VP1, small t-antigen, large T-antigen, exon 6 and 7 containing TrkA mRNAs, and exon 6 and 7 skipped $\Delta$ exon 6-7 TrkAIII mRNA and $18 \mathrm{~S}$ rRNA, as described previously [28]. Primer selection was dictated by the reported poor quality of degraded RNAs purified from FFPE tissues [31]. For semiquantitative densitometric analysis, triplicated RT-PCR products for TrkAIII and 18S rRNA were amplified from individual tissue samples, compared within the same 1\% agarose gel, digitally photographed and analysed using Image J software (ImageJ bundled with Java 1.8.0_172) [34]. Intergel comparisons were made using common $18 \mathrm{~S}$ rRNA RT-PCR product and DNA ladder standards, as previously described [28].

Furthermore, due to limited quantity and difficulty in extracting proteins from FFPE MCC tissues, evidence for TrkA protein isoform(s) expression and activation (TrkA Y490 tyrosine phosphorylation) was approximated in serial FFPE MCC tissue by indirect IF using TrkA carboxyl terminal-domain (C14, Santa Cruz Inc, Santa Cruz, CA, USA) and Y490 tyrosine-phosphorylated TrkA (pY490 TrkA, Cell Signaling, CA, USA) antibodies, as previously described [28,29].

The effect of chemotherapy on MCPyV, TrkA and $\Delta$ exon 6-7 TrkAIII mRNA expression and on TrkA- and Y490-phosphorylated TrkA isoform IF immunoreactivity was assessed by comparing FFPE tissues from the MCCs obtained prechemotherapy and in postchemotherapeutic recurrent MCCs from the same individual.

A fresh metastatic MCPyV-positive MCC tissue sample, surgically removed from one patient in the cohort, was used to extract good quality RNA for RT-PCR reactions, as previously described [29], in order to provide evidence of MCPyV gene VP-1, small $t$-antigen and large T-antigen mRNA and $18 \mathrm{~S}$ rRNA expression, using previously described primer sets [28], and for fully spliced TrkA, $\Delta$ exon 6-7 TrkAIII and additional TrkA isoforms, using primer sets spanning TrkA exons 1 to 17 (forward primer 5'-ATGCTGCGAGGCGGACGGCGC-3' and reverse primer 5' -CTAGCCCAGG ACATCCAGGTA- $3^{\prime}$ ), TrkA exons 1 to 8 (forward primer $5^{\prime}$-ATGCTGCGAGGCGGACGGCGC-3' and reverse primer $5^{\prime}$-GGAGGCCTGGCCGAAGGGGTT-3'), TrkA exons 10-17 (forward primer 5'-AACCCCTTCGGCCAGGCCTCC-3' and reverse primer 5'-CTAGCCCAGGACATCC AGGTA-3'), TrkA exon 6-7 (forward primer 5' - AGCCACGGTGATGAAATCTGGGGGTC T-3' and reverse primer 5'-TTGACCTGAACAGAGACCTCTGC-3') and the $\Delta$ exon 6-7 TrkAIII-specific primer set (forward primer 5'-AATGCCAGCTGTGTCCCG-3' and reverse primer 5'-TGGTC TCATTGAGCACGGAG-3') [35]. Gel extraction of ethidium bromide-stained RT-PCR products was performed using a QIAquick gel extraction kit, as described by the manufacturer (Qiagen, Milan, IT). Protein extracts from the fresh nonfixed MCC were subjected to Western blotting using an anti-TrkA antibody (C14, Santa Cruz Inc., Santa Cruz, CA, USA) that recognises both fully spliced TrkA and alternatively spliced $\Delta$ exon 6-7 TrkAIII [29] and were compared to fully spliced TrkA and $\Delta$ exon 6-7 TrkAIII proteins ( $5 \mu \mathrm{g})$, extracted from stable-transfected TrkA and $\Delta$ exon 6-7 TrkAIII SH-SY5Y cells [29].

\subsection{Statistical Analysis}

Statistical analyses were performed using STATA software (version 14, Stata Corp, College Station, TX, USA). For clinical data, continuous variables are expressed as median with interquartile range (iqr). For molecular data, continuous variables are displayed as the mean \pm standard deviation (SD). For both categories, qualitative data are expressed as frequency or percentage.

\section{Conclusions}

In conclusion and in pursuit of future therapeutic improvements for the treatment of MCC, we (i) stress the importance of multidisciplinary treatment, including surgical excisions and repetitive locoregional chemotherapy, not only for optimising local disease control [36] and enhancing overall 
survival [17-25] but also for palliative care, considering the psychological importance of surgical tumour removal; (ii) suggest that sofosbuvir/daclastavir therapy should be investigated as a potential indirect inhibitor of MCPyV oncogenic activity; (iii) confirm that oncogenic alternative $\Delta$ exon 6-7 TrkAIII splicing not only occurs in MCPyV-positive MCC but also in metastatic disease, focusing attention on this novel potential MCPyV oncogenic mechanism as a possible therapeutic target. We propose that detection alternative $\Delta$ exon 6-7 TrkAIII identifies an MCC subgroup that may benefit from novel inhibitors of MCPyV T-antigen and $\Delta$ exon 6-7 TrkAIII expression or clinically approved Trk kinase inhibitors such as larotrectinib and entrectinib, known to inhibit mutation and deletion-activated TrkA oncogenes and to elicit durable responses in a wide range of advanced-stage TrkA-fusion oncogene-driven cancers [37-39], supporting the call for a large multicentre study.

Author Contributions: S.G. and M.C., performed the surgical procedures; S.G., A.R.F., and A.R.M. designed this study; D.S. and A.C., analysed the clinical data; E.R., G.B., and G.F. were responsible for medical oncology; A.C. was responsible for histopathology; L.A.C., M.S., V.Z., A.R.F., A.R.M., and R.M., performed the molecular analyses; R.M. and A.R.M. performed the indirect immunofluorescence; S.G. and A.R.M. drafted the manuscript; G.F. and A.R.F. revised and validated the manuscript. All authors have read and agreed to the published version of the manuscript.

Funding: This research received no external funding.

Conflicts of Interest: The authors declare no conflict of interest.

\section{Abbreviations}

\begin{tabular}{|c|c|}
\hline bp & base pair \\
\hline CLND & complete lymph node dissection \\
\hline CD56 & neural cell adhesion molecule \\
\hline $\mathrm{CT}$ & computerised tomography \\
\hline EpCAM & epithelial cell adhesion molecule \\
\hline FDA & food and drug administration \\
\hline FFPE & formalin-fixed paraffin-embedded \\
\hline IF & indirect immunofluorescence \\
\hline $\operatorname{IgG1}$ & immunoglobulin G1 \\
\hline $\mathrm{INF} \gamma$ & interferon gamma \\
\hline ILI & isolated limb infusion \\
\hline ILP & isolated limb perfusion \\
\hline IPLP & isolated pelvic and limb perfusion \\
\hline MCCs & Merkel cell carcinomas \\
\hline MCPyV & Merkel cell polyomavirus \\
\hline NB & neuroblastoma \\
\hline NCCN & National Comprehensive Cancer Network \\
\hline NIH3T3 & mouse embryonic fibroblast cells \\
\hline OS & overall survival \\
\hline PD-1 & programmed cell death protein 1 \\
\hline PD-L1 & programmed death-ligand 1 \\
\hline RNAs & ribonucleic acids \\
\hline RT & radiotherapy \\
\hline RT-PCR & real-time polymerase chain reaction \\
\hline SNB & sentinel lymph node biopsy \\
\hline SD & standard deviation \\
\hline SV40 & simian vacuolating 40 polyomavirus \\
\hline TNF & tumour necrosis factor \\
\hline TrkA & tropomyosin-related kinase $\mathrm{A}$ \\
\hline USA & United States of America \\
\hline UV & ultraviolet \\
\hline VEGFR & vascular endothelial growth factor receptor \\
\hline WSE & wide surgical excisions \\
\hline WGSE & wide gluteal surgical excision \\
\hline SDi & stable disease \\
\hline PFS & Progression-free survival \\
\hline
\end{tabular}




\section{References}

1. Pulitzer, M. Merkel cell carcinoma. Surg. Pathol. Clin. 2017, 10, 399-408.

2. Clarke, C.A.; Robbins, H.A.; Tatalovich, Z.; Lynch, C.F.; Pawlish, K.S.; Finch, J.L.; Hernandez, B.Y.; Fraumeni, J.F., Jr.; Madeleine, M.M.; Engels, E.A. Risk of Merkel cell carcinoma after solid organ transplantation. J. Natl. Cancer Inst. 2015, 107, dju382. [CrossRef]

3. Amaral, T.; Leiter, U.; Garbe, C. Merkel cell carcinoma: Epidemiology, pathogenesis, diagnosis and therapy. Rev. Endocr. Metab. Disord. 2017, 18, 517-532.

4. Gonzalez-Vela, M.D.; Curiel-Olmo, S.; Derdak, S.; Beltran, S.; Santibañez, M.; Martínez, N.; Castillo-Trujillo, A.; Gut, M.; Sánchez-Pacheco, R.; Almaraz, C.; et al. Shared oncogenic pathways implicated in both virus-positive and UV-induced Merkel cell carcinomas. J. Investig. Dermatol. 2017, 137, 197-206.

5. Harms, P.W.; Vats, P.; Verhaegen, M.E.; Robinson, D.R.; Wu, Y.M.; Dhanasekaran, S.M.; Palanisamy, N.; Siddiqui, J.; Cao, X.; Su, F.; et al. The distinctive mutational spectra of polyomavirus-negative Merkel cell carcinoma. Cancer Res. 2015, 75, 3720-3727.

6. Bichakjian, C.K.; Olencki, T.; Aasi, S.Z.; Alam, M.; Andersen, J.S.; Blitzblau, R.; Bowen, G.M.; Contreras, C.M.; Daniels, G.A.; Decker, R.; et al. Merkel Cell Carcinoma, Version 1.2018. Clinical Practice Guidelines in Oncology. J. Natl. Compr. Cancer Netw. 2018, 16, 742-774.

7. Jouary, T.; Leyral, C.; Dreno, B.; Doussau, A.; Sassolas, B.; Beylot-Barry, M.; Renaud-Vilmer, C.; Guillot, B.; Bernard, P.; Lok, C.; et al. Adjuvant prophylactic regional radiotherapy versus observation in stage I Merkel cell carcinoma: A multicentric prospective randomized study. Ann. Oncol. 2012, 23, 1074-1080.

8. Poulsen, M. Radiation therapy rather than surgery for Merkel Cell Carcinoma: The advantages of radiation therapy. Int. J. Radiat. Oncol. Biol. Phys. 2018, 100, 14-15.

9. Harms, K.L.; Healy, M.A.; Nghiem, P.; Sober, A.J.; Johnson, T.M.; Bichakjian, C.K.; Wong, S.L. Analysis of prognostic factors from 9387 Merkel cell carcinoma cases forms the basis for the new 8th edition AJCC staging system. Ann. Surg. Oncol. 2016, 23, 3564-3571.

10. Lebbe, C.; Becker, J.C.; Grob, J.J.; Malvehy, J.; Del Marmol, V.; Pehamberger, H.; Peris, K.; Saiag, P.; Middleton, M.R.; Bastholt, L.; et al. Diagnosis and treatment of Merkel Cell Carcinoma. European consensus-based interdisciplinary guideline. Eur. J. Cancer 2015, 51, 2396-2403.

11. Iyer, J.G.; Blom, A.; Doumani, R.; Lewis, C.; Tarabadkar, E.S.; Anderson, A.; Ma, C.; Bestick, A.; Parvathaneni, U.; Bhatia, S.; et al. Response rates and durability of chemotherapy among 62 patients with metastatic Merkel cell carcinoma. Cancer Med. 2016, 5, 2294-2301.

12. Nghiem, P.T.; Bhatia, S.; Lipson, E.J.; Kudchadkar, R.R.; Miller, N.J.; Annamalai, L.; Berry, S.; Chartash, E.K.; Daud, A.; Fling, S.P.; et al. PD-1 blockade with pembrolizumab in advanced merkel-cell carcinoma. N. Engl. J. Med. 2016, 374, 2542-2552.

13. Kaufman, H.L.; Russell, J.; Hamid, O.; Bhatia, S.; Terheyden, P.; D'Angelo, S.P.; Shih, K.C.; Lebbé, C.; Linette, G.P.; Milella, M.; et al. Avelumab in patients with chemotherapy refractory metastatic Merkel cell carcinoma: A multicentre, single-group, open-label, phase 2 trial. Lancet Oncol. 2016, 17, 1374-1385.

14. Barkdull, S.; Brownell, I. PD-L1 blockade with avelumab: A new paradigm for treating Merkel cell carcinoma. Cancer Biol. Ther. 2017, 18, 937-939.

15. Tarabadkar, E.S.; Thomas, H.; Blom, A.; Parvathaneni, U.; Olencki, T.; Nghiem, P.; Bhatia, S. Clinical benefit from Tyrosine kinase inhibitors in metastatic Merkel cell carcinoma: A case series of 5 patients. Am. J. Case Rep. 2018, 19, 505-511.

16. Ollier, J.; Kervarrec, T.; Samimi, M.; Benlalam, H.; Aumont, P.; Vivien, R.; Touzé, A.; Labarrière, N.; Vié, H.; Clémenceau, B. Merkel cell carcinoma and cellular cytotoxicity: Sensitivity to cellular lysis and screening for potential target antigens suitable for antibody-dependent cellular cytotoxicity. Cancer Immunol. Immunother. 2018, 67, 1209-1219.

17. Gupta, A.; Heinzman, S.; Levine, E.A. Successful treatment of in-transit metastases from Merkel's cell carcinoma with isolated hyperthermic limb perfusion. South. Med. J. 1998, 91, 289-292.

18. Thiels, C.A.; Gonzalez, A.B.; Gray, R.J.; Jakub, J.W. Isolated limb perfusion in Merkel cell carcinoma offers high rate of complete response and durable local-regional control: Systematic review and institutional experience. J. Surg. Oncol. 2016, 114, 187-192.

19. Zeitouni, N.C.; Giordano, C.N.; Kane, J.M., 3rd. In-transit Merkel cell carcinoma treated with isolated limb perfusion or isolated limb infusion: A case series of 12 patients. Dermatol. Surg. 2011, 37, 357-364. 
20. Turaga, K.K.; Beasley, G.M.; Kane, J.M., 3rd; Delman, K.A.; Grobmye, S.R.; Gonzalez, R.J.; Letson, G.D.; Cheong, D.; Tyler, D.S.; Zager, J. Limb preservation with isolated limb infusion for locally advanced nonmelanoma cutaneous and soft-tissue malignant neoplasms. Arch. Surg. 2011, 146, 870-875.

21. Steinman, J.; Ariyan, C.; Rafferty, B.; Brady, M.S. Factors associated with response, survival, and limb salvage in patients undergoing isolated limb infusion. J. Surg. Oncol. 2014, 109, 405-409.

22. Olieman, A.F.T.; Lienard, D.; Eggermont, A.M.; Kroon, B.B.; Lejeune, F.J.; Hoekstra, H.J.; Koops, H.S. Hyperthermic isolated limb perfusion with tumor necrosis factor, interferon gamma, and melphalan for locally advanced nonmelanoma skin tumors of the extremities. Arch. Surg. 1999, 134, 303-307.

23. van Veenendaal, L.M.; Madu, M.F.; Tesselaar, M.E.T.; Verhoef, C.; Grünhagen, D.J.; van Akkooi, A.C.J. Efficacy of isolated limb perfusion (ILP) in patients withMerkel cell carcinoma (MCC): A multicenter experience. Eur. J. Surg. Oncol. 2017, 43, 2157-2162.

24. Guadagni, S.; Chiominto, A.; Mackay, A.R.; Farina, A.R.; Cappabianca, L.; Puccica, I.; Valiyevae, S.; Clementi, M. Advanced Merkel Cell Carcinoma of the lower extremity treated with surgery and Isolated Pelvic and Limb Perfusion using Melphalan: A case of unexpected long-term survival. Int. J. Surg. Case Rep. 2019, 61, 4-8.

25. Kroon, H.M.; Thompson, J.F. Letter reply to Efficacy of isolated limb perfusion (ILP) in patients with Merkel cell carcinoma: A multicenter experience. Eur. J. Surg. Oncol. 2019, 45, 1121-1123.

26. Guadagni, S.; Kanavos, E.; Schietroma, M.; Fiorentini, G.; Amicucci, G. Selected hypoxic stop-flow perfusions: Indication and limits. Tumori 2006, 92, 402-406.

27. Guadagni, S.; Palumbo, G.; Fiorentini, G.; Clementi, M.; Marsili, L.; Giordano, A.V.; Masedu, F.; Valenti, M. Surgical versus percutaneous Isolated Pelvic Perfusion (IPP) for advanced melanoma: Comparison in terms of melphalan pharmacokinetic pelvic bio-availability. BMC Res. Notes 2017, 10, 411. [CrossRef]

28. Cappabianca, L.; Guadagni, S.; Maccarone, R.; Sebastiano, M.; Chiominto, A.; Farina, A.R.; Mackay, A.R. A pilot study of alternative TrkAIII splicing in Merkel cell carcinoma: A potential oncogenic mechanism and novel therapeutic target. J. Exp. Clin. Cancer Res. 2019, 38, 424. [CrossRef]

29. Tacconelli, A.; Farina, A.R.; Cappabianca, L.; Desantis, G.; Tessitore, A.; Vetuschi, A.; Sferra, R.; Rucci, N.; Argenti, B.; Screpanti, I.; et al. TrkA alternative splicing: A regulated tumor-promoting switch in human neuroblastoma. Cancer Cell. 2004, 6, 347-360.

30. Farina, A.R.; Cappabianca, L.; Ruggeri, P.; Gneo, L.; Pellegrini, C.; Fargnoli, M.-C.; Mackay, A.R. The oncogenic neurotrophin receptor tropomyosin-related kinase variant, TrkAIII. J. Exp. Clin. Cancer Res. 2018, 37, 119. [CrossRef]

31. Wimmer, I.; Troscher, A.R.; Brunner, F.; Rubino, S.J.; Bien, C.G.; Weiner, H.L.; Lassmann, H.; Bauer, J. Systematic evaluation of RNA quality, microarray data reliability and pathway analysis in fresh, fresh frozen and formalin-fixed paraffin-embedded tissue samples. Sci. Rep. 2018, 8, 6351. [CrossRef]

32. Qi, Y.; Liao, D.; Mei, D.; Zhang, Y.; Liu, Y. Elevated neutrophil-to-lymphocyte ratio is associated with poor outcomes for melanoma patients treated with PD-1 inhibitor or chemotherapy in a Chinese population. Front. Oncol. 2020, 10, 1752. [CrossRef]

33. Agha, R.; Abdall-Razak, A.; Crossley, E.; Dowlut, N.; Iosifidis, C.; Mathew, G.; STROCSS Group. The STROCSS 2019 Guideline: Strengthening the Reporting of Cohort Studies in Surgery. Int. J. Surg. 2019, 72, 156-165.

34. Schindelin, J.; Rueden, C.T.; Hiner, M.C.; Eliceiri, K.W. The ImageJ ecosystem: An open platform for biomedical image analysis. Mol. Reprod. Dev. 2015, 82, 518-529.

35. Lebedev, T.D.; Vagapova, E.R.; Popenko, V.I.; Leonova, O.G.; Spirin, P.V.; Prassolov, V.S. Two receptors, two isoforms, two cancers: Comprehensive analysis of KIT and TrkA expression in neuroblastoma and acute myeloid leukemia. Front. Oncol. 2019, 9, 1046. [CrossRef]

36. Aliberti, C.; Carandina, R.; Sarti, D.; Pizzirani, E.; Ramondo, G.; Cillo, U.; Guadagni, S.; Fiorentini, G. Transarterial chemoembolization with DC Bead LUMI ${ }^{\mathrm{TM}}$ radiopaque beads for primary liver cancer treatment: Preliminary experience. Future Oncol. 2017, 13, 2243-2252.

37. Lang, A.M.; Lo, H.-W. Inhibiting TRK proteins in clinical cancer therapy. Cancers 2018, 10, 105. [CrossRef] 
38. Drilon, A.; Laetsch, T.W.; Kummer, S.; DuBois, S.G.; Lassen, U.N.; Demetri, G.D.; Nathenson, M.; Doebele, R.C.; Farago, A.F.; Alberto, S.; et al. Efficacy of Larotrectinib in Trk fusion-positive cancer in adults and children. N. Eng. J. Med. 2018, 378, 731-739.

39. Cocco, E.; Scalriti, M.; Drilon, A. NTRK fusion positive cancers and Trk inhibitor therapy. Nat. Rev. Clin. Oncol. 2018, 15, 731-747.

Publisher's Note: MDPI stays neutral with regard to jurisdictional claims in published maps and institutional affiliations.

(C) 2020 by the authors. Licensee MDPI, Basel, Switzerland. This article is an open access article distributed under the terms and conditions of the Creative Commons Attribution (CC BY) license (http://creativecommons.org/licenses/by/4.0/). 